\section{Cushing, Harvey Williams}

M. Bidlingmaier

Medizinische Klinik und Poliklinik IV, Klinikum der

Universität München, München, Deutschland

Lebensdaten Geboren am 8. April 1869 in Cleveland, Ohio; gestorben am 7. Oktober 1939 in New Haven, Connecticut. Amerikanischer Neurochirurg. Harvey Cushing entstammte einer puritanischen Arztfamilie. College in Yale, seit 1891 Medizinstudium an der Harvard Medical School in Boston, unter anderem bei William Osler. Nach Abschluss des Studiums und einem Jahr als Assistenzarzt am Massachusetts General Hospital Wechsel an das Johns Hopkins Hospital in Baltimore. Von 1912 bis zur Emeritierung 1932 Professor für Chirurgie am neu gegründeten Peter Bent Brigham Hospital in Boston (Harvard Medical School).

Verdienste Cushing ist hauptsächlich bekannt als einer der bedeutendsten Vertreter der Neurochirurgen des 20. Jahrhunderts. Neben den dokumentierten 2023 Operationen von Hirntumoren trug er durch bahnbrechende Entwicklungen zur Verbesserungen chirurgischer und neurochirurgischer
Operationstechniken bei. U. a. gehen die Einführung von Silber-Clips zur Blutungskontrolle sowie die Elektrokauterisation auf Cushing zurück. Er war auch einer der ersten, die eine durchgängige Messung und Dokumentation von Vitalparametern zur Überwachung der Narkose einführten (Narkoseprotokoll). Durch Cushings Wirken konnte die Mortalität vieler chirurgischer Eingriffe drastisch reduziert werden. Auf ihn geht auch die Erstbeschreibung des durch einen ACTH-produzierenden Tumors des Hypophysenvorderlappens verursachten Hyperkortisolismus zurück. Der mit dem Kortisolexzess assoziierte Symptomenkomplex wurde 1943 auf Anregung des Endokrinologen Albright „Morbus Cushing“ genannt. Von Cushing stammen auch wichtige Arbeiten zur histologischen Klassifikation von Hypophysentumoren und zur Akromegalie.

\section{Literatur}

Bliss M (2005) Harvey Cushing: a life in surgery. University of Toronto Press, Toronto, Canada

Cushing H (1912) The pituitary body and its disorders: clinical states produced by disorders of the hypophysis cerebri. J. B. Lippincott Company, Philadelphia/London 